\title{
Stability of Linear Dynamic Systems on Time Scales
}

\author{
Sung Kyu Choi, ${ }^{1}$ Dong Man Im, ${ }^{2}$ and Namjip Koo ${ }^{1}$ \\ ${ }^{1}$ Department of Mathematics, Chungnam National University, Daejeon 305-764, South Korea \\ ${ }^{2}$ Department of Mathematics Education, Cheongju University, Cheongju 360-764, South Korea \\ Correspondence should be addressed to Namjip Koo, njkoo@math.cnu.ac.kr
}

Received 31 May 2007; Revised 23 February 2008; Accepted 16 March 2008

Recommended by Martin Bohner

We examine the various types of stability for the solutions of linear dynamic systems on time scales and give two examples.

Copyright (C) 2008 Sung Kyu Choi et al. This is an open access article distributed under the Creative Commons Attribution License, which permits unrestricted use, distribution, and reproduction in any medium, provided the original work is properly cited.

\section{Introduction}

Continuous and discrete dynamical systems have a number of significant differences mainly due to the topological fact that in one case the time scale $\mathbb{T}=\mathbb{R}$, real numbers, and the corresponding trajectories are connected while in other case $\mathbb{T}=\mathbb{Z}$, integers, they are not. The correct way of dealing with this duality is to provide separate proofs. All investigations on the two time scales show that much of the analysis is analogous but, at the same time, usually additional assumptions are needed in the discrete case in order to overcome the topological deficiency of lacking connectedness. Thus, we need to establish a theory that allows us to handle systematically both time scales simultaneously. To create the desired theory requires to setup a certain structure of $\mathbb{T}$ which is to play the role of the time scale generalizing $\mathbb{R}$ and $\mathbb{Z}$. Furthermore, an operation on the space of functions from $\mathbb{T}$ to the state space has to be defined generalizing the differential and difference operations. This work was initiated by Hilger [1] in the name of "calculus on measure chains or time scales."

In this paper, we examine the various types of stability-stability, uniform stability, asymptotic stability, strong stability, restrictive stability, and so forth, for the solutions of linear dynamic systems on time scales and give two examples.

\section{Preliminaries on dynamic systems}

We mention without proof several foundational definitions and results in the calculus on time scales from an excellent introductory text by Bohner and Peterson [2]. A time scale $\mathbb{T}$ is 
a nonempty closed subset of $\mathbb{R}$, and the forward jump operator $\sigma: \mathbb{T} \rightarrow \mathbb{T}$ is defined by

$$
\sigma(t)=\inf \{s \in \mathbb{T}: s>t\},
$$

(supplemented by $\inf \varnothing=\sup \mathbb{T}$ ), while the graininess $\mu: \mathbb{T} \rightarrow \mathbb{R}_{+}$is given by

$$
\mu(t)=\sigma(t)-t
$$

If $\mathbb{T}$ has a left-scattered maximum $m$, then $\mathbb{T}^{\kappa}:=\mathbb{T} \backslash\{m\}$ and otherwise $\mathbb{T}^{\mathcal{K}}:=\mathbb{T}$. A function $f: \mathbb{T} \rightarrow \mathbb{R}$ is called differentiable at $t \in \mathbb{T}^{\mathcal{K}}$, with (delta) derivative $f^{\Delta}(t) \in \mathbb{R}$ if given $\varepsilon>0$, there exists a neighborhood $U$ of $t$ such that, for all $s \in U$,

$$
\left|f^{\sigma}(t)-f(s)-f^{\Delta}(t)[\sigma(t)-s]\right| \leq \varepsilon|\sigma(t)-s|
$$

where $f^{\sigma}=f \circ \sigma$.

Some basic properties of delta derivatives are given in the following [3-5]:

(i) If $f$ is differentiable at $t \in \mathbb{T}^{\mathcal{K}}$, then

$$
f^{\sigma}(t)=f(t)+\mu(t) f^{\Delta}(t)
$$

(ii) If both $f$ and $g$ are differentiable at $t \in \mathbb{T}^{\kappa}$, then the product $f g$ is also differentiable at $t \in \mathbb{T}^{\mathcal{K}}$ with

$$
(f g)^{\Delta}(t)=f^{\Delta}(t) g(t)+f^{\sigma}(t) g^{\Delta}(t)=f(t) g^{\Delta}(t)+f^{\Delta}(t) g^{\sigma}(t)
$$

A function $f: \mathbb{T} \rightarrow \mathbb{R}$ is said to be $r d$-continuous (denoted by $f \in C_{\mathrm{rd}}(\mathbb{T}, \mathbb{R})$ ) if

(i) $f$ is continuous at every right-dense point $t \in \mathbb{T}$,

(ii) $\lim _{s \rightarrow t^{-}} f(s)$ exists and is finite at every left-dense point $t \in \mathbb{T}$.

A function $g: \mathbb{T} \rightarrow \mathbb{R}$ is called an antiderivative of $f$ on $\mathbb{T}$ if it is differentiable on $\mathbb{T}$ and satisfies $g^{\Delta}(t)=f(t)$ for $t \in \mathbb{T}^{\kappa}$. In this case, we define

$$
\int_{a}^{t} f(s) \Delta s=g(t)-g(a),
$$

where $t, a \in \mathbb{T}$.

The norm of an $n \times n$ matrix $M$ is defined to be

$$
|M|=\max _{1 \leq j \leq n}\left|M^{j}\right|
$$

where $M^{j}$ is the $j$ th column of $M$.

Let $M_{n}(\mathbb{R})$ be the set of all $n \times n$ matrices over $\mathbb{R}$. The class of all rd-continuous and regressive functions $A: \mathbb{T} \rightarrow M_{n}(\mathbb{R})$ is denoted by

$$
C_{\mathrm{rd}} \mathcal{R}\left(\mathbb{T}, M_{n}(\mathbb{R})\right)
$$


Here, a matrix-valued function $A$ is called regressive provided:

$$
I+\mu(t) A(t) \quad \text { is invertible } \forall t \in \mathbb{T},
$$

where $I$ is the identity matrix.

Definition 2.1. Let $t_{0} \in \mathbb{T}$. The unique matrix-valued solution of the IVP

$$
Y^{\Delta}=A(t) Y, \quad Y\left(t_{0}\right)=I,
$$

where $A \in C_{\mathrm{rd}} \mathcal{R}\left(\mathbb{T}, M_{n}(\mathbb{R})\right)$, is called the matrix exponential function and it is denoted by $e_{A}\left(t, t_{0}\right)$.

\section{Stability of linear dynamic systems}

We consider the dynamic system

$$
x^{\Delta}=F(t, x), \quad x\left(t_{0}\right)=x_{0},
$$

where $F \in C_{\text {rd }}\left(\mathbb{T} \times \mathbb{R}^{n}, \mathbb{R}^{n}\right)$ with $F(t, 0)=0$, and $x^{\Delta}$ is the delta derivative of $x: \mathbb{T} \rightarrow \mathbb{R}^{n}$ with respect to $t \in \mathbb{T}$. We assume that the solutions of (3.1) exist and are unique for $t \geq t_{0}$, and $\mathbb{T}$ is unbounded above.

We give the definitions about the various types of stability for the solutions of (3.1).

Definition 3.1. The solution $x$ of (3.1) is said to be stable if, for each $\varepsilon>0$, there exists a $\delta=$ $\delta(\varepsilon)>0$ such that, for any solution $\bar{x}(t)=x\left(t, t_{0}, \bar{x}_{0}\right)$ of $(3.1)$, the inequality $\left|\bar{x}_{0}-x_{0}\right|<\delta$ implies $|\bar{x}(t)-x(t)|<\varepsilon$ for all $t \geq t_{0} \in \mathbb{T}$.

Definition 3.2. The solution $x$ of (3.1) is said to be uniformly stable if, for each $\varepsilon>0$, there exists a $\delta=\delta(\varepsilon)>0$ such that, for any solution $\bar{x}(t)=x\left(t, t_{0}, \bar{x}_{0}\right)$ of $(3.1)$, the inequalities $t_{1} \geq t_{0}$ and $\left|\bar{x}\left(t_{1}\right)-x\left(t_{1}\right)\right|<\delta$ imply $|\bar{x}(t)-x(t)|<\varepsilon$ for all $t \geq t_{1} \in \mathbb{T}$.

Definition 3.3. The solution $x$ of (3.1) is said to be asymptotically stable if it is stable and there exists a $\delta_{0}>0$ such that $\left|\bar{x}_{0}-x_{0}\right|<\delta_{0}$ implies $|\bar{x}(t)-x(t)| \rightarrow 0$ as $t \rightarrow \infty$.

The following notion of strong stability is due to Ascoli [6].

Definition 3.4. The solution $x$ of (3.1) is said to be strongly stable if, for each $\varepsilon>0$, there exists a $\delta=\delta(\varepsilon)>0$ such that, for any solution $\bar{x}(t)$ of (3.1), the inequalities $t_{1} \geq t_{0}$ and $\left|\bar{x}\left(t_{1}\right)-x\left(t_{1}\right)\right|<\delta$ imply $|\bar{x}(t)-x(t)|<\varepsilon$ for all $t \geq t_{0} \in \mathbb{T}$.

For the other types of stability, that is, $h$-stability, we refer to [7].

We note that the stability of any solution of (3.1) is closely related to the stability of the null solution of the corresponding variational equation. Therefore, we will discuss the stability of linear dynamic system.

We consider the linear homogeneous dynamic system

$$
x^{\Delta}=A(t) x(t), \quad x\left(t_{0}\right)=x_{0},
$$

where $A \in C_{\mathrm{rd}} \mathcal{R}\left(\mathbb{T}, M_{n}(\mathbb{R})\right)$. 
It follows that any solution of the linear dynamic system is (uniformly, strongly, asymptotically) stable if and only if the same holds for the zero solution of (3.2). We say that (3.2) is (uniformly, strongly, asymptotically stable) stable if so is the null solution of (3.2). See [8].

Firstly, we show that the stability for solutions of (3.2) is equivalent to the boundedness.

Theorem 3.5. Equation (3.2) is stable if and only if all solutions of (3.2) are bounded for all $t \geq t_{0} \in \mathbb{T}$.

Proof. Suppose that (3.2) is stable. Since the trivial solution $x\left(t, t_{0}, 0\right)=0$ is stable, given any $\varepsilon>0$, there exists a $\delta>0$ such that $\left|x_{0}\right|<\delta$ implies $\left|x\left(t, t_{0}, x_{0}\right)\right|<\varepsilon$. Note that $\left|x\left(t, t_{0}, x_{0}\right)\right|=$ $\left|e_{A}\left(t, t_{0}\right) x_{0}\right|<\varepsilon$ for all $t \geq t_{0} \in \mathbb{T}$. Now, let $x_{0}$ be a vector of length $\delta / 2$ in the $j$ th direction for $j=1,2, \ldots, n$. Then, $\left|e_{A}\left(t, t_{0}\right) x_{0}\right|=\left|x_{j}(t)\right|(\delta / 2)<\varepsilon$, where $x_{j}(t)$ is the $j$ th column of $e_{A}\left(t, t_{0}\right)$. Thus, we have

$$
\left|e_{A}\left(t, t_{0}\right)\right|=\max _{1 \leq j \leq n}\left|x_{j}(t)\right|<\frac{2 \varepsilon}{\delta}
$$

Consequently, for any solution $x\left(t, t_{0}, x_{0}\right)$ of (3.2),

$$
\left|x\left(t, t_{0}, x_{0}\right)\right|=\left|e_{A}\left(t, t_{0}\right) x_{0}\right|<\frac{2 \varepsilon}{\delta}\left|x_{0}\right|
$$

It follows that all solutions of (3.2) are bounded.

For the converse, we note that all solutions of (3.2) are bounded if and only if there exists a positive constant $M$ such that

$$
\left|e_{A}\left(t, t_{0}\right)\right| \leq M, \quad t \geq t_{0} .
$$

It follows from $x(t)=e_{A}\left(t, t_{0}\right) x_{0}$ that (3.2) is stable. This completes the proof.

In [9, Theorem 2.1], DaCunha obtained the following characterization of uniform stability by means of the operator norm. It is not difficult to prove this result by using the maximum norm.

Theorem 3.6. Equation (3.2) is uniformly stable if and only if there exists a positive constant $\gamma$ such that

$$
\left|e_{A}\left(t, t_{0}\right)\right| \leq \gamma,
$$

for all $t \geq t_{0}$ with $t, t_{0} \in \mathbb{T}$.

The following is the characterization of strong stability for linear dynamic system (3.2). Note that its continuous version was presented in [10].

Theorem 3.7. Equation (3.2) is strongly stable if and only if there exists a positive constant $M$ such that

$$
\left|e_{A}\left(t, t_{0}\right)\right| \leq M, \quad\left|e_{A}^{-1}\left(t, t_{0}\right)\right| \leq M, \quad t \geq t_{0} \in \mathbb{T}
$$

where $e_{A}\left(t, t_{0}\right)$ is a matrix exponential function of (3.2). 
Proof. Suppose that (3.7) holds. For any given $\varepsilon>0$, we can choose $\delta=\varepsilon / 2 M^{2}$ such that for any $t_{1} \geq t_{0},\left|x_{1}\right|=\left|x\left(t_{1}, t_{0}, x_{0}\right)\right|<\delta$. Then, we have

$$
\begin{aligned}
\left|x\left(t, t_{0}, x_{0}\right)\right| & =\left|e_{A}\left(t, t_{0}\right) x_{0}\right| \\
& =\left|e_{A}\left(t, t_{0}\right) e_{A}^{-1}\left(t_{1}, t_{0}\right) x_{1}\right| \\
& \leq\left|e_{A}\left(t, t_{0}\right)\right|\left|e_{A}^{-1}\left(t_{1}, t_{0}\right)\right|\left|x_{1}\right| \\
& \leq M^{2}\left|x_{1}\right| \\
& <\varepsilon, \quad t \geq t_{0} \in \mathbb{T} .
\end{aligned}
$$

Hence, (3.2) is strongly stable.

Conversely, if (3.2) is strongly stable, then we have

$$
\left|e_{A}\left(t, t_{0}\right) e_{A}^{-1}\left(t_{1}, t_{0}\right) x_{1}\right|<\varepsilon, \quad t \geq t_{0} \in \mathbb{T}
$$

whenever $t_{1} \geq t_{0}$ and $\left|x_{1}\right|<\delta$ holds. Since $x_{1}$ is arbitrary, we have

$$
\left|e_{A}\left(t, t_{0}\right) e_{A}^{-1}\left(t_{1}, t_{0}\right)\right|<M
$$

where $M=2 n \varepsilon / \delta$. It is clear that $\delta$, and hence $M$, is independent of $t_{0}$ and $t_{1}$ as well as of $t$. Putting $t_{1}=t_{0}$ and $t=t_{0}$, we obtain the result.

Example 3.8 (see [8]). (i) The system $x^{\Delta}=0$ is strongly stable, but it is not asymptotically stable.

(ii) The system $x^{\Delta}=\alpha x$ with $-1<\alpha \mu(t)<0$ is asymptotically stable, but it is not strongly stable.

Restrictive stability in [10] is related to strong stability, and we obtain their equivalence for (3.2) as a consequence of Theorem 3.7.

Definition 3.9. System (3.2) is said to be restrictively stable if it is stable and its adjoint system

$$
x^{\Delta}=-A^{*}(t) x^{\sigma}
$$

where $A^{*}$ denotes the conjugate transpose of $A$, is stable.

Remark 3.10. We note that (3.2) is strongly stable if and only if it is restrictively stable.

Definition 3.11. System (3.2) is said to be reducible (or reducible to zero), if there exists $L \in C_{\mathrm{rd}}^{1}\left(\mathbb{T}, M_{n}(\mathbb{R})\right)$ which is bounded together with its inverse $L^{-1}(t)$ on $\mathbb{T}_{t_{0}}$ such that $L^{\sigma^{-1}}(t) A(t) L(t)-L^{\sigma^{-1}}(t) L^{\Delta}(t)$ is a constant (or zero) matrix on $\mathbb{T}_{t_{0}}$. Here, $\mathbb{T}_{t_{0}}=\left[t_{0}, \infty\right) \cap \mathbb{T}$, and the set of all functions $L: \mathbb{T} \rightarrow M_{n}(\mathbb{R})$ that are differentiable and whose derivative is rd-continuous is denoted by

$$
C_{\mathrm{rd}}^{1}\left(\mathbb{T}, M_{n}(\mathbb{R})\right)
$$

Theorem 3.12. System (3.2) is restrictively stable if and only if it is reducible to zero. 
Proof. Let $e_{A}\left(t, t_{0}\right)$ be a matrix exponential function of (3.2). Suppose that (3.2) is restrictively stable. Then, there exists a positive constant $M$ such that

$$
\left|e_{A}\left(t, t_{0}\right)\right| \leq M, \quad\left|e_{A}^{-1}\left(t, t_{0}\right)\right| \leq M, \quad t \geq t_{0} \in \mathbb{T},
$$

by means of Theorem 3.7. Consider the transformation $x=e_{A}\left(t, t_{0}\right) y$. Then, it follows that

$$
A(t) e_{A}\left(t, t_{0}\right) y=x^{\Delta}=e_{A}^{\Delta}\left(t, t_{0}\right) y+e_{A}\left(\sigma(t), t_{0}\right) y^{\Delta}
$$

Hence, we obtain

$$
y^{\Delta}=\left[e_{A}^{-1}\left(\sigma(t), t_{0}\right) A(t) e_{A}\left(t, t_{0}\right)-e_{A}^{-1}\left(\sigma(t), t_{0}\right) e_{A}^{\Delta}\left(t, t_{0}\right)\right] y=0,
$$

since $e_{A}^{-1}\left(\sigma(t), t_{0}\right) A(t) e_{A}\left(t, t_{0}\right)-e_{A}^{-1}\left(\sigma(t), t_{0}\right) e_{A}^{\Delta}\left(t, t_{0}\right)=0$. This implies that (3.2) is reducible to zero.

For the converse, suppose that there exists $L \in C_{\mathrm{rd}}^{1}\left(\mathbb{T}, M_{n}(\mathbb{R})\right)$ such that

$$
L^{-1}(\sigma(t)) A(t) L(t)-L^{-1}(\sigma(t)) L^{\Delta}(t)=0 .
$$

Then, we have $L^{\Delta}=A(t) L(t)$. Thus, $L(t)$ is a matrix exponential function of (3.2). Since $L(t)$ and $L^{-1}(t)$ are bounded for all $t \geq t_{0} \in \mathbb{T}$, the proof is complete.

Theorem 3.13. If (3.2) is stable and reducible on a time scale $\mathbb{T}$ with the constant graininess, then it is uniformly stable.

Proof. Since (3.2) is reducible, we have

$$
y^{\Delta}=B y(t)
$$

where $B=L^{-1}(\sigma(t)) A(t) L(t)-L^{-1}(\sigma(t)) L^{\Delta}(t)$ by the transformation $x=L(t) y$. Let $e_{A}\left(t, t_{0}\right)$ be a matrix exponential function of (3.2). The stability of (3.2) implies the boundednesss of $e_{A}\left(t, t_{0}\right)$. Let $e_{A}\left(t, t_{0}\right)=L(t) e_{B}\left(t, t_{0}\right)$, where $e_{B}\left(t, t_{0}\right)$ is a matrix exponential function of (3.17). Then, we have

$$
e_{B}\left(t, t_{0}\right)=L^{-1}(t) e_{A}\left(t, t_{0}\right), \quad e_{A}^{-1}\left(t, t_{0}\right)=e_{B}^{-1}\left(t, t_{0}\right) L^{-1}(t),
$$

and hence the boundedness of $e_{A}\left(t, t_{0}\right)$ implies the boundedness of $e_{B}\left(t, t_{0}\right)$ since $L^{-1}(t)$ is bounded. Thus, (3.17) is stable and, in fact, is uniformly stable. Hence, it is clear that

$$
\left|e_{B}\left(t, t_{0}\right) e_{B}^{-1}\left(\tau, t_{0}\right)\right| \leq M
$$

for some positive constant $M$ and all $t_{0} \leq \tau \leq t \in \mathbb{T}$. Therefore,

$$
\begin{aligned}
\left|e_{A}\left(t, t_{0}\right) e_{A}^{-1}\left(\tau, t_{0}\right)\right| & =\left|L(t) e_{B}\left(t, t_{0}\right) e_{B}^{-1}\left(\tau, t_{0}\right) L^{-1}(\tau)\right| \\
& \leq|L(t)|\left|e_{B}\left(t, t_{0}\right) e_{B}^{-1}\left(\tau, t_{0}\right)\right|\left|L^{-1}(\tau)\right| \\
& \leq N
\end{aligned}
$$

for some positive constant $N$ and all $t_{0} \leq \tau \leq t \in \mathbb{T}$. Consequently, (3.2) is uniformly stable. 
The continuous versions of Theorems 3.12 and 3.13 are presented in (3.9.v) and (3.9.vi) in [10], respectively.

Remark 3.14. It does not hold in general that every stable linear homogeneous system with constant coefficient matrix on a time scale $\mathbb{T}$ is uniformly stable.

Corollary 3.15. If (3.11) is stable and $q(t)=\lambda_{1} \oplus \lambda_{2} \oplus \cdots \oplus \lambda_{n}>0$ with the eigenvalues $\lambda_{i}(1 \leq j \leq n)$ of $A(t)$, then it is restrictively stable.

Proof. It follows from the stability of (3.2) that $e_{A}\left(t, t_{0}\right)$ is bounded for all $t \geq t_{0} \in \mathbb{T}$. Furthermore, by Liouville's formula [11], we have

$$
\operatorname{det} e_{A}\left(t, t_{0}\right)=e_{q}\left(t, t_{0}\right) \operatorname{det} e_{A}\left(t_{0}, t_{0}\right) \geq d>0, \quad t \in \mathbb{T},
$$

where $d$ is a positive constant. Thus, from

$$
e_{A}^{-1}\left(t, t_{0}\right)=\frac{\operatorname{adj} e_{A}\left(t, t_{0}\right)}{\operatorname{det} e_{A}\left(t, t_{0}\right)}
$$

it is clear that $e_{A}^{-1}\left(t, t_{0}\right)$ is bounded for all $t \geq t_{0} \in \mathbb{T}$. The proof is complete.

Remark 3.16. Pötzsche et al. [12] proved a necessary and sufficient condition for the exponential stability of time-variant linear systems on time scales in terms of the eigenvalues of the system matrix. They used a representation formula for the transition matrix of Jordan reducible systems in the regressive case.

Remark 3.17. In summary, the following assertions are all equivalent [13, Theorem 4.2].

(i) System (3.2) is strongly stable.

(ii) There exists a positive constant $M$ such that

$$
\left|e_{A}\left(t, t_{0}\right)\right| \leq M, \quad\left|e_{A}^{-1}\left(t, t_{0}\right)\right| \leq M, \quad t \geq t_{0} \in \mathbb{T} .
$$

(iii) Adjoint system (3.11) of (3.2) is strongly stable.

(iv) System (3.2) is restrictively stable.

(v) System (3.2) is reducible to zero.

It is widely known that the stability characteristics of a nonautonomous linear system of differential or difference equations can be characterized completely by a corresponding autonomous linear system by the Lyapunov transformation. DaCunha and Davis in [14] gave a definition of the Lyapunov transformation as follows.

Let $L \in C_{\mathrm{rd}}^{1}\left(\mathbb{T}, M_{n}(\mathbb{R})\right)$. The Lyapunov transformation is an invertible matrix-valued function $L$ with the property that, for some positive $\eta, \rho \in \mathbb{R}$,

$$
|L(t)| \leq \rho, \quad \operatorname{det} L(t) \geq \eta
$$

for all $t \in \mathbb{T}$. 
Remark 3.18. Note that the boundedness of the coefficient matrices is not preserved by the Lyapunov transformation $L$ in the case of the time scales with right-dense point [13]. This can be seen by considering the time scale $\mathbb{T}=\mathbb{R}$ and the Lyapunov transformation:

$$
L(t)=\left[\begin{array}{cc}
\sin \left(t^{2}\right) & \cos \left(t^{2}\right) \\
-\cos \left(t^{2}\right) & \sin \left(t^{2}\right)
\end{array}\right] .
$$

It shows that the coefficient matrices $A$ and $B$ satisfy

$$
B(t)=L^{-1}(\sigma(t)) A(t) L(t)-L^{-1}(\sigma(t)) L^{\Delta}(t),
$$

where $A(t)=\left[\begin{array}{ll}1 & 0 \\ 0 & 1\end{array}\right]$, and $B(t)=\left[\begin{array}{cc}1 & 2 t \\ -2 t & 1\end{array}\right]$.

Now, we consider the linear dynamic system

$$
z^{\Delta}=G(t) z(t), \quad z\left(t_{0}\right)=z_{0},
$$

and its perturbed system

$$
w^{\Delta}=G(t) w(t)+F(t) w(t), \quad w\left(t_{0}\right)=w_{0},
$$

where $G, F \in C_{\text {rd }} \mathcal{R}\left(\mathbb{T}, M_{n}(\mathbb{R})\right)$.

The following theorem means that the strong stability for the system (3.27) is equivalent to that of (3.2).

Lemma 3.19 (see [14, Theorem 3.8]). Suppose that $L \in C_{\mathrm{rd}}^{1}\left(\mathbb{T}, M_{n}(\mathbb{R})\right)$ is invertible for all $t \in \mathbb{T}$, and $A \in C_{\mathrm{rd}}\left(\mathbb{T}, M_{n}(\mathbb{R})\right)$ is regressive. Then, the transformation matrix for the system

$$
Z^{\Delta}=G(t) Z(t), \quad Z(\tau)=I,
$$

where

$$
G(t)=L^{\sigma^{-1}}(t) A(t) L(t)-L^{\sigma^{-1}}(t) L^{\Delta}(t),
$$

is given by

$$
e_{G}(t, \tau)=L^{-1}(t) e_{A}(t, \tau) L(\tau)
$$

for all $t, \tau \in \mathbb{T}$.

The regressiveness of $G(t)$ in (3.30) is preserved by the Lyapunov transformation in the following lemma.

Lemma 3.20. Suppose that $L \in C_{\mathrm{rd}}^{1}\left(\mathbb{T}, M_{n}(\mathbb{R})\right)$ is the transformation matrix for all $t \in \mathbb{T}$. Then $A$ is regressive if and only if $G$ is also regressive.

Proof. We see that for every right-scattered point $t \in \mathbb{T}^{\kappa}$, the following identity holds:

$$
I+\mu(t) G(t)=L^{-1}(\sigma(t))[I+\mu(t) A(t)] L(t) .
$$

This completes the proof. 
Theorem 3.21. Suppose that $z(t)=L^{-1}(t) x(t)$ is a Lyapunov transformation. Then (3.2) is strongly stable if and only if (3.27) is strongly stable.

Proof. Suppose that (3.2) is strongly stable. Then, there exists a constant $M>0$ such that

$$
\left|e_{A}\left(t, t_{0}\right)\right| \leq M, \quad\left|e_{A}^{-1}\left(t, t_{0}\right)\right| \leq M, \quad t \geq t_{0} \in \mathbb{T} .
$$

By using Lemma 3.19, we have

$$
\begin{aligned}
& \left|e_{G}\left(t, t_{0}\right)\right|=\left|L^{-1}(t) e_{A}\left(t, t_{0}\right) L\left(t_{0}\right)\right| \leq \rho^{2} M=\widehat{M}, \\
& \left|e_{G}^{-1}\left(t, t_{0}\right)\right|=\left|L^{-1}\left(t_{0}\right) e_{A}^{-1}\left(t, t_{0}\right) L(t)\right| \leq \rho^{2} M=\widehat{M}, \quad t \geq t_{0} \in \mathbb{T} .
\end{aligned}
$$

Hence, (3.27) is strongly stable.

The converse holds similarly.

If we assume that the perturbing term $F$ is absolutely integrable, then we obtain the uniform stability for the perturbed system (3.28) when system (3.2) is strongly stable.

Theorem 3.22. Suppose that $z(t)=L^{-1}(t) x(t)$ is a Lyapunov transformation and there exists a $\beta>0$ such that for all $t_{0} \in \mathbb{T}$ :

$$
\int_{t_{0}}^{\infty}|F(s)| \Delta s \leq \beta .
$$

If (3.2) is strongly stable, then (3.28) is uniformly stable.

Proof. It follows from Theorem 3.21 that (3.27) is strongly stable. Then, there exists a positive constant $M$ such that

$$
\left|e_{G}\left(t, t_{0}\right)\right| \leq M, \quad\left|e_{G}^{-1}\left(t, t_{0}\right)\right| \leq M \quad \text { for } t \geq t_{0} \in \mathbb{T} .
$$

For any $t_{0}$ and $w\left(t_{0}\right)=w_{0}$, the solution $w(t)$ of (3.28) satisfies

$$
w(t)=e_{G}\left(t, t_{0}\right) w_{0}+\int_{t_{0}}^{t} e_{G}(t, \sigma(s)) F(s) w(s) \Delta s .
$$

By taking the norms of both sides of (3.37), we have

$$
|w(t)| \leq M\left|w_{0}\right|+M^{2} \int_{t_{0}}^{t}|F(s)||w(s)| \Delta s, \quad t \geq t_{0} .
$$

In view of Gronwall's inequality [15], we obtain

$$
\begin{aligned}
|w(t)| \leq M\left|w_{0}\right| e_{M^{2}|F(s)|}\left(t, t_{0}\right) & \begin{cases}M\left|w_{0}\right| \exp \left(\int_{t_{0}}^{t} \frac{\log \left(1+\mu(s) M^{2}|F(s)|\right)}{\mu(s)} \Delta s\right) & \text { if } \mu(t) \neq 0, \\
M\left|w_{0}\right| \exp \left(\int_{t_{0}}^{t} M^{2}|F(s)| \Delta s\right) & \text { if } \mu(t)=0\end{cases} \\
& \leq M\left|w_{0}\right| \exp \left(\int_{t_{0}}^{t} M^{2}|F(s)| \Delta s\right) \\
& \leq M\left|w_{0}\right| \exp \left(\int_{t_{0}}^{\infty} M^{2}|F(s)| \Delta s\right),
\end{aligned}
$$


for all $t \geq t_{0} \in \mathbb{T}$. Thus

$$
|w(t)| \leq d\left|w_{0}\right|, \quad t \geq t_{0}
$$

where $d=M e^{M^{2} \beta}$. Hence, (3.28) is uniformly stable.

\section{Examples}

In this section, we give two examples about the various types of stability for solutions of linear dynamic systems on time scales in [16].

Example 4.1. To illustrate Theorem 3.7, we consider the linear dynamic system

$$
x^{\Delta}=A(t) x=\left(\begin{array}{cc}
\frac{-e^{-t}}{2+e^{-t}} & 0 \\
0 & 0
\end{array}\right) x, \quad x\left(t_{0}\right)=x_{0}, \quad t \geq t_{0} \in \mathbb{T},
$$

where $A(t)=\left(\begin{array}{cc}-e^{-t} /\left(2+e^{-t}\right) & 0 \\ 0 & 0\end{array}\right)$. If $\mu(t)<2 e^{t}+1$ for all $t \in \mathbb{T}$, then (4.1) is strongly stable.

Remark 4.2. We give some remarks about Example 4.1.

(1) If $\mathbb{T}=\mathbb{R}$, then $e_{A}(t, 0)$ of linear differential system $x^{\Delta}=x^{\prime}=A(t) x$ is given by

$$
e_{A}(t, 0)=\left(\begin{array}{cc}
\frac{2+e^{-t}}{3} & 0 \\
0 & 1
\end{array}\right), \quad t \in \mathbb{R}
$$

(2) If $\mathbb{T}=h \mathbb{Z}$ with the positive constant $h<2 e^{t}+1$ for all $t \in h \mathbb{Z}$, then $e_{A}(t, 0)$ of linear difference system

$$
x^{\Delta}=\frac{x(t+h)-x(t)}{h}=A(t) x
$$

is given by

$$
e_{A}(t, 0)=\left(\begin{array}{cc}
\prod_{\tau=0}^{t-h}\left(1-\frac{e^{-\tau}}{2+e^{-\tau}} h\right) & 0 \\
0 & 1
\end{array}\right), \quad t \in h \mathbb{Z}
$$

(3) If $\mathbb{T}=q^{\mathbb{N}_{0}}$ with the constant $q>1$, then $e_{A}(t, 1)$ of $q$-difference system

$$
x^{\Delta}=\frac{x(q t)-x(t)}{(q-1) t}=A(t) x
$$

is given by

$$
e_{A}(t, 1)=\left(\prod_{\tau \in \mathbb{T} \cap(0, t)}\left(\begin{array}{cc}
\left.1+\frac{(1-q) \tau e^{-\tau}}{2+e^{-\tau}}\right) & 0 \\
0 & 1
\end{array}\right), \quad t \in q^{\mathbb{N}_{0}} .\right.
$$


Sung Kyu Choi et al.

(4) If $\mathbb{T}=\mathbb{P}_{1,1}=\cup_{k=0}^{\infty}[2 k, 2 k+1]$ and $p(t)=-e^{-t} /\left(2+e^{-t}\right)$, then $e_{A}(t, 0)$ of linear dynamic system

$$
x^{\Delta}= \begin{cases}x^{\prime}=A(t) x(t) & \text { if } t \in[2 k, 2 k+1), \\ \Delta x(t)=A(t) x(t) & \text { if } t=2 k+1\end{cases}
$$

is given by

$$
e_{A}(t, 0)=\left(\begin{array}{cc}
e_{p}(t, 0) & 0 \\
0 & 1
\end{array}\right), \quad t \in \mathbb{T}=\mathbb{P}_{1,1}
$$

Example 4.3. We consider the linear dynamic system

$$
x^{\Delta}=A(t) x=\left(\begin{array}{cc}
0 & 0 \\
0 & -2
\end{array}\right) x, \quad x\left(t_{0}\right)=x_{0}, \quad t \geq t_{0} \in \mathbb{T},
$$

where $A(t)=\left(\begin{array}{cc}0 & 0 \\ 0 & -2\end{array}\right)$. If $\mu(t) \neq 1 / 2$ for all $t \in \mathbb{T}$, then the matrix exponential function $e_{A}\left(t, t_{0}\right)$ of (4.9) is given by

$$
e_{A}\left(t, t_{0}\right)=\left(\begin{array}{cc}
1 & 0 \\
0 & e_{-2}\left(t, t_{0}\right)
\end{array}\right), \quad t \geq t_{0} \in \mathbb{T} .
$$

We see that the generalized exponential function $e_{-2}(t, 0)$ is given by

$$
e_{-2}(t, 0)= \begin{cases}e^{-2 t}, & t \in \mathbb{R}, \\ (1-2 h)^{t / h}, & t \in h \mathbb{Z}, \\ \prod_{\tau \in q^{\mathbb{N} 0} \cap[0, t)}(1+(1-q) 2 \tau), & t \in q^{\mathbb{N}_{0},} \\ \left(-e^{2}\right)^{k} e^{-2 t}, & t \in \bigcup_{k=0}^{\infty}[2 k, 2 k+1],\end{cases}
$$

respectively. Thus, we obtain the following results for (4.9) and $e_{-2}\left(t, t_{0}\right)$.

(1) If $\mathbb{T}=\mathbb{R}$, then (4.9) is uniformly stable but not strongly stable.

(2) If $\mathbb{T}=\mathbb{Z}$, then (4.9) is strongly stable but not asymptotically stable.

(3) If $\mathbb{T}=h \mathbb{Z}$ with $0<h<1$ and $h \neq 1 / 2$, then (4.9) is neither asymptotically stable nor strongly stable. However, $e_{-2}\left(t, t_{0}\right)$ goes to zero as $t \rightarrow \infty$.

(4) If $\mathbb{T}=h \mathbb{Z}$ with $h>1$, then (4.9) is neither asymptotically stable nor strongly stable.

(5) If $\mathbb{T}=q^{\mathbb{N}_{0}}$ with $q>3 / 2$, then (4.9) is unbounded and $e_{-2}\left(t, t_{0}\right)$ is oscillatory.

(6) If $\mathbb{T}=\cup_{k=0}^{\infty}[2 k, 2 k+1]$, then (4.9) is bounded and $e_{-2}\left(t, t_{0}\right)$ goes to zero as $t \rightarrow \infty$.

\section{Acknowledgments}

The authors are thankful to the anonymous referees for their valuable comments and corrections to improve this paper. This work was supported by the Korea Research Foundation Grant founded by the Korea Government (MOEHRD) (KRF-2005-070-C00015). 


\section{References}

[1] S. Hilger, "Analysis on measure chains-a unified approach to continuous and discrete calculus," Results in Mathematics, vol. 18, no. 1-2, pp. 18-56, 1990.

[2] M. Bohner and A. Peterson, Dynamic Equations on Time Scales. An Introduction with Applications, Birkhäuser, Boston, Mass, USA, 2001.

[3] R. Agarwal, M. Bohner, D. O'Regan, and A. Peterson, "Dynamic equations on time scales: a survey," Journal of Computational and Applied Mathematics, vol. 141, no. 1-2, pp. 1-26, 2002.

[4] M. Bohner and R. Hering, "Perturbations of dynamic equations," Journal of Difference Equations and Applications, vol. 8, no. 4, pp. 295-305, 2002.

[5] V. Lakshmikantham, S. Sivasundaram, and B. Kaymakcalan, Dynamic Systems on Measure Chains, Kluwer Academic Publishers, Dordrecht, The Netherlands, 1996.

[6] G. Ascoli, "Osservazioni sopra alcune questioni di stabilità. I," Atti della Accademia Nazionale dei Lincei. Rendiconti. Classe di Scienze Fisiche, Matematiche e Naturali, vol. 9, pp. 129-134, 1950.

[7] S. K. Choi, N. Koo, and D. M. Im, " $h$-stability for linear dynamic equations on time scales," Journal of Mathematical Analysis and Applications, vol. 324, no. 1, pp. 707-720, 2006.

[8] M. Rama Mohana Rao, Ordinary Differential Equations, Edward Arnold, London, UK, 1981.

[9] J. J. DaCunha, "Stability for time varying linear dynamic systems on time scales," Journal of Computational and Applied Mathematics, vol. 176, no. 2, pp. 381-410, 2005.

[10] L. Cesari, Asymtotic Behavior and Stability Properties in Ordinary Differential Equations, Springer, Berlin, Germany, 2nd edition, 1963.

[11] V. Cormani, "Liouville's formula on time scales," Dynamic Systems and Applications, vol. 12, no. 1-2, pp. 79-86, 2003.

[12] C. Pötzsche, S. Siegmund, and F. Wirth, "A spectral characterization of exponential stability for linear time-invariant systems on time scales," Discrete and Continuous Dynamical Systems, vol. 9, no. 5, pp. 1223-1241, 2003.

[13] B. Aulbach and C. Pötzsche, "Reducibility of linear dynamic equations on measure chains," Journal of Computational and Applied Mathematics, vol. 141, no. 1-2, pp. 101-115, 2002.

[14] J. J. DaCunha and J. M. Davis, "Periodic linear systems: Lyapunov transformations and a unified Floquet theory for time scales," preprint, 2005.

[15] P. Agarwal, M. Bohner, and A. Peterson, "Inequalities on time scales: a survey," Mathematical Inequalities \& Applications, vol. 4, no. 4, pp. 535-557, 2001.

[16] S. K. Choi and N. Koo, "On the stability of linear dynamic systems on time scales," to appear in Journal of Difference Equations and Applications. 